\title{
The Difference between Sacubitril Valsartan and Valsartan on Vascular Endothelial Function, APN, MMP-9, and BNP Levels in Patients with Hypertension and Chronic Heart Failure
}

\author{
Haiping Du $\mathbb{D},{ }^{1}$ Xiao Li $\mathbb{D}^{2},{ }^{2}$ Weifang Zhao $\mathbb{D}^{3},{ }^{3}$ and Ning Jiang $\mathbb{D}^{4}$ \\ ${ }^{1}$ Department of Cardiology (I), East Hospital, Yantaishan Hospital, Yantai 264000, Shandong, China \\ ${ }^{2}$ Department of Cardiology (II), The Third People's Hospital of Qingdao, Qingdao University, Qingdao 266041, Shandong, China \\ ${ }^{3}$ Cardiac Function Examination Room, Affiliated Qingdao Central Hospital, Qingdao University, Qingdao 266042, \\ Shandong, China \\ ${ }^{4}$ Department of Hypertension, Jinan Municipal Hospital of Traditional Chinese Medicine, Jinan 250012, Shandong, China
}

Correspondence should be addressed to Ning Jiang; jiangning@jnzyhospital.cn

Received 24 December 2021; Accepted 21 January 2022; Published 18 February 2022

Academic Editor: Bhagyaveni M.A

Copyright (C) 2022 Haiping Du et al. This is an open access article distributed under the Creative Commons Attribution License, which permits unrestricted use, distribution, and reproduction in any medium, provided the original work is properly cited.

Background. Sacubitril valsartan and valsartan are the first new drugs approved for angiotensin receptor neprilysin lysine inhibitors (ARNIs) in outpatients with chronic heart failure (CHF) and hypertension. Compared with enalapril, sacubitril valsartan and valsartan have been shown to reduce the mortality and morbidity of cardiovascular diseases. However, there is little actual evidence regarding the efficacy of ARNIs in hypertensive patients with CHF. Methods. From January 2019 to January 2021,60 patients with hypertension and chronic heart failure were diagnosed and treated in our hospital. The patients were randomly divided into an observation group and a control group, with 30 cases in each group. The control group was given valsartan, the observation group was given sacubitril valsartan, and both groups were treated for six months. The endothelium-dependent vasodilation (EDD) function of the brachial artery and serum nitric oxide (NO), endothelin-1 (ET-1), carotid artery intima-media thickness, and glomerular filtration, excess rate (eGFR), and left ventricular ejection fraction (LVEF) were compared between the two groups of patients before and after treatment. The serum adiponectin (APN), matrix metalloproteinase-9 (MMP-9), and brain natriuretic peptide (BNP) levels were compared before and after treatment. Results. The total effective rate of treatment in the research group was higher than that in the control group $(P<0.05)$. After treatment, the cardiac function indexes LVESD and LVEDD of the two groups of patients were lower than before treatment, and LVEF was higher than before treatment, and the improvement rate of the treatment group was better than that of the control group $(P<0.05)$. After treatment, the serum APN of the two groups was higher than before treatment, the levels of MMP-9 and BNP were lower than before treatment, and the improvement rate of patients in the treatment group was better than that of patients in the control group $(P<0.05)$. There was no statistically significant in the levels of EDD, NO, and ET-1 of the two groups of patients before treatment $(P<0.05)$. After treatment, compared with the control group, the EDD function and NO level of the research group were significantly increased $(P<0.05)$, and the level of ET-1 was significantly reduced $(P<0.05)$. There was no statistically significant difference in carotid artery intima-media thickness, glomerular filtration rate, and left ventricular ejection fraction before and after treatment in the two groups $(P<0.05)$. Conclusion. In the treatment of hypertension and chronic heart failure, sacubitril valsartan can improve the clinical symptoms of patients to the greatest extent and can significantly improve the levels of LVEF, LVEDD, NT-proBNP, heart function, and other indicators. Sacubitril valsartan can increase serum APN levels, reduce MMP-9 and BNP levels, and have good clinical effects. Sacubitril valsartan has a protective effect on the vascular endothelial function of patients with hypertension and $\mathrm{CHF}$. However, these results need to be confirmed in studies involving more subjects and require longer follow-up times. 


\section{Introduction}

Chronic heart failure ( $\mathrm{CHF}$ ) is one of the common diseases in cardiology. Chronic heart failure is mostly caused by a variety of factors that lead to changes in the structure and function of the heart, causing ventricular contraction and/or diastolic dysfunction so that the cardiac output cannot meet the metabolic needs of the body, thereby causing cardiac circulatory disorders [1]. In recent years, due to changes in dietary structure, the number of people with high blood pressure in my country has gradually increased, reaching $27.1 \%$ [2], and long-term uncontrolled high blood pressure can lead to $\mathrm{CHF}$. At present, despite the continuous improvement of CHF treatment, its 5-year survival rate is still low $[3,4]$. In the occurrence and development of CHF, its vascular endothelial dysfunction and the disease itself are mutually causal. Endothelial dysfunction leads to an imbalance in the levels of NO and ET-1 released by endothelial cells, resulting in abnormal vasomotor function, which further leads to increased vascular resistance and increased cardiac afterload, thereby aggravating myocardial ischemia and hypoxia, pumping dysfunction, and forming a vicious circle [5-7].

An important feature of the pathogenesis of hypertension and chronic heart failure is the activation of the neurohormonal system, including the renin-angiotensinaldosterone system (RAAS) and the sympathetic nervous system (SNS). In the short term, the compensatory activation of these systems is conducive to increasing blood pressure and enhancing cardiac contractility, which stems from their evolutionary "biological protection" role. However, the continuous excessive activation of these systems often produces the opposite effect, inducing and aggravating cardiovascular disease and worsening heart failure due to fluid retention [8]. Based on the understanding of its mechanism, drugs that inhibit these neurohormonal systems have been shown to be used to treat cardiovascular diseases, such as hypertension and heart failure, and can reduce the mortality and hospitalization risk of heart failure by 37\% [9]. Therefore, it is particularly important to choose a reasonable and effective treatment plan for patients with hypertension and chronic heart failure.

Sacubitril valsartan and valsartan are angiotensin receptor-enkephalinase inhibitors (ARNI), which can simultaneously inhibit RAAS and regulate NPS, promote water and sodium excretion, dilate blood vessels, and antagonize sympathetic activity. At the same time, sacubitril valsartan and valsartan have anti-inflammatory, antiventricular remodeling, and antihypertensive effects, thereby improving the treatment effect and prognosis of patients [10-12]. Therefore, sacubitril valsartan and valsartan are approved as first-line treatments to reduce heart failure [13]. Clinical trials have confirmed the effects of sacubitril valsartan and valsartan in improving heart failure and revealed their potential in blood pressure control $[14,15]$. Studies have shown that it is easier to improve the prognosis in patients with $\mathrm{CHF}$ than angiotensin-converting enzyme inhibitors (ACEI) $[16,17]$. Sacubitril valsartan sodium is mainly used clinically to treat chronic heart failure with reduced ejection fraction. Sacubitril valsartan sodium inhibits enkephalinase through the active metabolite of the prodrug sacubitril and, at the same time, block the type 1 receptor of angiotensin II by valsartan, thereby improving the prognosis in patients with chronic heart failure [18].

This article explores 60 patients with hypertension and chronic heart failure, adopts the clinical efficacy of two treatments, provides a more clinical application basis for the adjustment of blood pressure level, and provides better guidance for clinical work. This study aims to further explore and analyze the effects of valsartan and valsartan treatment of sacubitril on vascular endothelial function, APN, MMP-9, and BNP levels in patients with hypertension and chronic heart failure based on previous research.

\section{Material and Methods}

2.1. Research Object. A total of 60 patients with hypertension and chronic heart failure who were diagnosed and treated in our hospital from January 2019 to January 2021 were selected and randomly divided into a research group and a control group. The patients were randomly grouped, and the patients were numbered according to the sample size, from 1 to 60, and random numbers were generated using SPSS26.0. Starting from any number in the random number table, take a random number for each research subject in sequence along the same direction. Thirty patients with a larger random number were used as the research group, and 30 patients with a smaller random number were used as the control group. This study was conducted after being approved by the ethics committee of our hospital.

Inclusion criteria were as follows: meeting the diagnostic criteria for hypertension; patients aged $\geq 18$ years; patients with chronic heart failure whose heart function is graded IIIV by NYHA; patients who have good compliance and can be followed up in time. The patients and their families have been informed of the purpose and methods of this study, and their right to informed consent is reserved.

Exclusion criteria were as follows: patients who are allergic to all the ingredients of the drug or its active ingredients (sacubitril valsartan) or any excipients; patients with angioedema (whether hereditary or idiopathic); patients with severe liver function impairment; patients with biliary cirrhosis and cholestasis; patients with severe renal impairment (GFR $<30 \mathrm{ml} / \mathrm{min}$ ), serum potassium $>5.4 \mathrm{mmol} /$ $\mathrm{L}$; patients in the second and third trimesters of pregnancy; diabetic patients who also used aliskiren; patients with systemic diseases such as tumors and immune systems; patients who refused to continue to participate in the study and who were lost to follow-up.

\subsection{Research Methods}

2.2.1. Treatment Methods. All patients undergo routine treatment and care for chronic heart failure and hypertension, including limiting salt intake, proper diuresis, oxygen inhalation, correcting electrolytes, and application of drugs ( $\beta$-blockers, vasodilators). On the basis of conventional treatment, patients in the control group chose 
valsartan capsules (National Medicine Standard H20040217, the specification is $80 \mathrm{mg} /$ capsule) $80 \mathrm{mg} /$ time, twice a day. The research group added sacubitril and valsartan sodium tablets (Beijing Novartis Pharmaceutical Co., Ltd., specification $100 \mathrm{mg}$, National Standard H20170343) $50 \mathrm{mg} /$ time on the basis of conventional treatment, twice a day. According to the tolerance, sacubitril and valsartan sodium tablets are increased to $100 \mathrm{mg} /$ time, two times a day, orally. The blood pressure control goals of the two groups of patients were systolic blood pressure $<130 \mathrm{mmHg}$ and diastolic blood pressure $<80 \mathrm{mmHg}$. If the blood pressure control target cannot be achieved, additional drug doses or combined application of other drugs can be used if necessary.

\subsection{Evaluation of Detection Indicators and Clinical Efficacy}

\subsubsection{Criteria for Clinical Efficacy Evaluation.} Significantly effective: the clinical symptoms and signs of the patient such as dyspnea, fatigue, and edema were significantly improved compared to before treatment, and the NYHA cardiac function classification improved to 2 or more. Effective: the patient's clinical symptoms and signs such as dyspnea, fatigue, and edema improved compared with those before treatment, and the NYHA cardiac function classification improved by level 1. Ineffective: compared with the patients before treatment, the clinical symptoms and signs such as dyspnea, fatigue, and edema, and NYHA heart function classification did not improve or worsen compared with those before treatment.

$$
\text { Total effective rate }=\frac{(\text { marked effect }+ \text { effective })}{\text { total number of cases }} \times 100 \% \text {. }
$$

\subsubsection{Brachial Artery Endothelium-Dependent Vasodilation} Function (EDD). The baseline and follow-up examinations of all study subjects were performed by fixed ultrasound staff who had received uniform training. All patients were placed supine, had an empty stomach, rested for 30 minutes, and underwent color Doppler ultrasound examination. The upper arm was fully exposed, and the distance between the anterior and posterior intima of the brachial artery was recorded as the inner diameter (D0). Place a blood pressure cuff closer to the ultrasound probe and inflate it until the arterial systolic pressure is $\geq 30 \mathrm{mmHg}$. The arterial blood flow was blocked for about 5 minutes, then the cuff was quickly loosened to induce reactive hyperemia, and the inner diameter of the brachial artery (D1) was measured again after 1 minute of deflation.

$$
\mathrm{EDD}=\frac{(D 1-D 0)}{D 0} \times 100 \% \text {. }
$$

2.3.3. Heart Function Test. All patients were tested by echocardiography (Shanghai Hanfei Medical Equipment Co., Ltd.). The changes of LVESD, LVEDD, CO, LVEF, and other indicators were observed before and after treatment in the two groups.

2.3.4. NO and Endothelin (ET-1) Expression Level. In total, $4 \mathrm{~mL}$ of fasting venous blood was collected, and the levels of NO and ET-1 were measured by the nitrate reductase method and radioimmunoassay. The operation methods are strictly in accordance with the instructions. The above indicators were measured and recorded before and after treatment.

2.3.5. Determination of Plasma NT-proBNP. An enzymelinked immunosorbent assay (ELISA) method was used to collect $2 \mathrm{ml}$ of fasting venous blood from the patient. The blood was placed in an anticoagulation test tube and a centrifuge with a rotation speed of $3000 \mathrm{r} / \mathrm{min}$ for 10 minutes of centrifugation was used. Plasma was separated and placed in a refrigerator at $-20{ }^{\circ} \mathrm{C}$ overnight. The detection kit was provided by Wuhan Elabscience Biotechnology Co., Ltd. (EEL-R3023). An automatic immunochemiluminescence instrument was used to detect plasma NT-proBNP levels. The correlation coefficient between NT-proBNP concentration and absorbance on the standard curve is $>0.995$, indicating that the quality control parameters are within the standard error.

2.3.6. Determination of APN and MMP-9 Levels. Serum adiponectin (APN) and matrix metalloproteinase-9 (MMP9) levels were drawn on fasting before and after treatment. Enzyme-linked immunosorbent assay was used to detect the levels of APN and MMP-9 in the two groups before and after treatment.

2.4. Statistical Methods. The detection index data of the two groups of patients are expressed as mean \pm standard deviation (SD). SPSS22.0 statistical analysis software was used for processing. Differences between different groups were compared by analysis of variance or chi-square test, and data between the same groups were tested by $t$-test. $P<0.05$ indicates that the difference is statistically significant. Each experiment was repeated three times.

\section{Results}

3.1. Baseline Characteristics of Patients. This study selected 60 patients with hypertension and chronic heart failure. There were 30 cases in the research group, including 21 male patients and 9 female patients, with an average age of $74.37 \pm 3.5$ years. There were 30 cases in the control group, including 19 males and 11 females, with an average age of $75.97 \pm 3.72$ years. In general data comparison, the two groups of patients are comparable, and the difference is not statistically significant $(P>0.05)$ (Table 1$)$.

3.2. Comparison of Clinical Effective Rate of Treatment between the Two Groups of Patients. In the research group, 2 cases were ineffective, 22 cases were effective, and 6 cases 
TABLE 1: Comparison of general information.

\begin{tabular}{|c|c|c|c|c|}
\hline Project & Research group & Control group & $t / x^{2}$ & $P$ \\
\hline Gender (male/female) & $21 / 9$ & $19 / 11$ & 0.3 & 0.58 \\
\hline Age (year) & $74.37 \pm 3.5$ & $75.97 \pm 3.72$ & 1.71 & 0.09 \\
\hline Mean arterial pressure $(\mathrm{mmHg})$ & $130.21 \pm 10.98$ & $128.45 \pm 11.15$ & 0.62 & 0.54 \\
\hline BMI $\left(\mathrm{kg} / \mathrm{m}^{2}\right)$ & $31.23 \pm 4.01$ & $30.11 \pm 4.25$ & 1.06 & 0.29 \\
\hline
\end{tabular}

were markedly effective. The total effective rate was $93.3 \%$ $(28 / 30)$. In the control group, 5 cases were ineffective, 15 cases were effective, and 10 cases were markedly effective. The effective rate was $83.3 \%(25 / 30)$.

3.3. Comparison of EDD Function between the Two Groups of Patients before and after Treatment. The difference in D0 between the two groups of patients after treatment was not statistically significant. Compared with the control group, the D1 $(5.30 \pm 1.85) \mathrm{mm}$ in the research group after treatment was significantly higher than before $(4.58 \pm 1.31)$ $(P<0.05)$. EDD $(11.83 \pm 2.81) \mathrm{mm}$ after treatment in the research group was significantly higher than before treatment $(9.23 \pm 2.17)(P<0.05)$, as shown in Figure 1 .

3.4. Heart Function Test. Before treatment, there was no significant difference in the levels of LVEDD, LVESD, and LVEF between the two groups of patients $(P>0.05)$ (Table 2). After treatment, the levels of heart function indexes LVEDD and LVESD of the two groups of patients were lower than before treatment $(P<0.05)$, LVEF levels were higher than before treatment, and the improvement of the research group was better than that of the control group, and the difference was statistically significant $(P<0.05)$ (Table 2).

3.5. Comparison of Plasma NO and ET-1 Levels before and after Treatment in the Two Groups. Compared with the control group, the NO $(97.03 \pm 7.53 \mu \mathrm{moL} / \mathrm{L})$ of the research group after treatment was significantly higher than that before treatment $(83.04 \pm 6.64 \mu \mathrm{moL})$. Compared with the control group, the ET-1 $(42.32 \pm 4.82 \mathrm{ng} / \mathrm{L})$ of the research group after treatment was significantly higher than that before treatment $(49.97 \pm 5.43 \mathrm{ng} / \mathrm{L})$ (Figure 2).

3.6. Determination of NT-proBNP, APN, and MMP-9 Levels before and after Treatment. By comparison, we found that the NT-proBNP level in the research group before treatment was $4858.82 \pm 512.34 \mathrm{pg} / \mathrm{ml}$, the control group was $4835.43 \pm 496.76 \mathrm{pg} / \mathrm{ml}$, and there was no statistical difference between the two groups. After treatment, the NTproBNP level in the research group was $985.35 \pm 312.38 \mathrm{pg} /$ $\mathrm{ml}$, and the control group was $1203.19 \pm 304.23 \mathrm{pg} / \mathrm{ml}$. After treatment, the levels of NT-proBNP in the two groups were reduced compared with the same group before treatment, and there was a statistical difference. After treatment, the NT-proBNP level of the research group was significantly lower than that of the control group, and there were significant differences, indicating that the improvement of the research group was more obvious than that of the control group (Figure 3(a)). It can be seen from Figures 3(b) and 3(c) that there was no statistically significant difference in the levels of serum APN and MMP-9 between the two groups before treatment $(P>0.05)$. After treatment, the serum APN of the two groups was higher than that before treatment, and the level of MMP-9 was lower than that before treatment, and the improvement of the treatment group was better than that of the control group $(P<0.05)$.

3.7. Comparison of Carotid Artery Intima-Media Thickness, Glomerular Filtration Rate, and Left Ventricular Ejection Fraction before and after Treatment between the Two Groups. The difference in carotid artery intima-media thickness, glomerular filtration rate, and left ventricular ejection fraction before and after treatment in the two groups was not statistically significant $(P>0.05)$ (Table 3$)$.

\section{Discussion}

Chronic heart failure $(\mathrm{CHF})$ is a clinical syndrome characterized by excessive activation of neuroendocrine hormones and ventricular remodeling [19]. Chronic heart failure is the main clinical manifestation of various causes of heart disease in the terminal stage, with high morbidity and mortality. The main drugs currently used to treat chronic heart failure include the traditional golden triangle, including $\beta$-receptor blockers, aldosterone receptor antagonists, and angiotensin-converting enzyme inhibitors (ACEI)/angiotensin receptor antagonists (ARB). Among them, ACEI/ARB and $\beta$-blockers can effectively reduce the mortality of patients and improve the prognosis of patients [20]. ACEI/ARB and $\beta$-blockers play a key role in chronic heart failure and improve the clinical course of the disease [21]. The cornerstone of the treatment of chronic heart failure is to inhibit the renin-angiotensin-aldosterone system (RAAS). In addition, inhibiting enkephalinase and increasing the level of the natriuretic peptide can also effectively inhibit the activation of the neuroendocrine system, and the combination of the two can better play a synergistic effect [22]. The new type of chronic heart failure treatment drug sacubitril valsartan is the only angiotensin receptorenkephalinase inhibitor (ARNI) used in clinical treatment. Sacubitril valsartan has a dual mechanism of action: not only can it inhibit enkephalinase, but also it antagonize angiotensin II receptors and have stronger antiproliferative and antifibrotic effects on cardiomyocytes [10].

Sacubitril valsartan is a new type of medicine for treating heart failure, mainly including sacubitril and valsartan. Sacubitril is an enkephalinase inhibitor, and enkephalinase is 


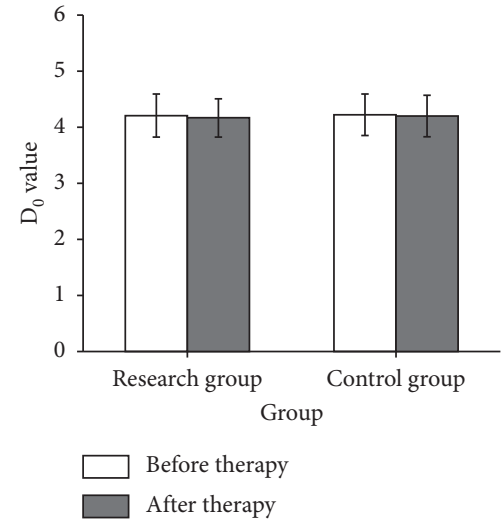

(a)

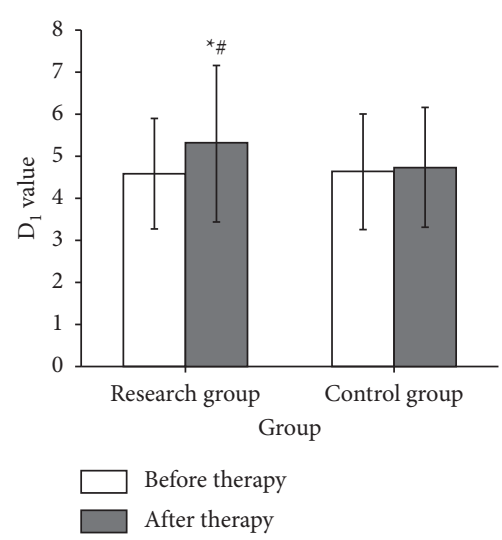

(b)

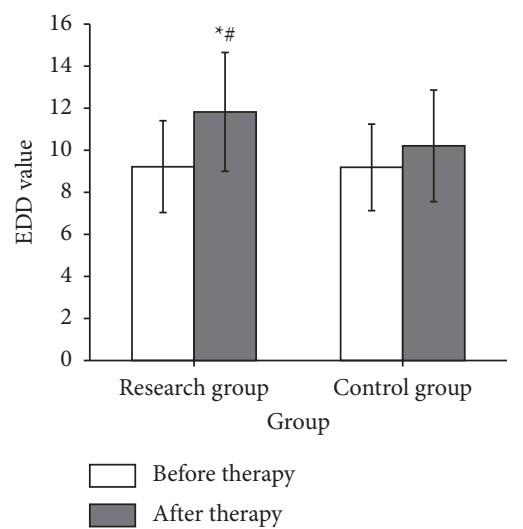

(c)

Figure 1: Comparison of EDD function between two groups of patients before and after treatment. Note. Compared with before treatment: ${ }^{*} P<0.05$; compared with the control group: ${ }^{\#} P<0.05$; (a) D0, (b) D1, and (c) EDD.

TABLE 2: Comparison of echocardiographic indicators before and after treatment between the two groups.

\begin{tabular}{|c|c|c|c|c|c|}
\hline Time & Index & Control group & Research group & $\mathrm{t}$ value & $P$ value \\
\hline \multirow{3}{*}{ Before treatment } & LVEDD $(\mathrm{mm})$ & $61.21 \pm 9.14$ & $62.76 \pm 9.32$ & 0.65 & 0.52 \\
\hline & LVESD (mm) & $41.89 \pm 7.14$ & $42.31 \pm 7.99$ & 0.21 & 0.83 \\
\hline & LVEF (\%) & $31.32 \pm 4.96$ & $30.04 \pm 5.03$ & 0.99 & 0.32 \\
\hline \multirow{3}{*}{ After treatment } & LVEDD (mm) & $57.79 \pm 6.87$ & $54.87 \pm 6.45$ & 1.70 & 0.09 \\
\hline & LVESD $(\mathrm{mm})$ & $37.85 \pm 5.42$ & $34.36 \pm 5.04$ & 2.58 & 0.01 \\
\hline & LVEF (\%) & $34.48 \pm 4.52$ & $36.88 \pm 5.02$ & 0.99 & 0.32 \\
\hline
\end{tabular}

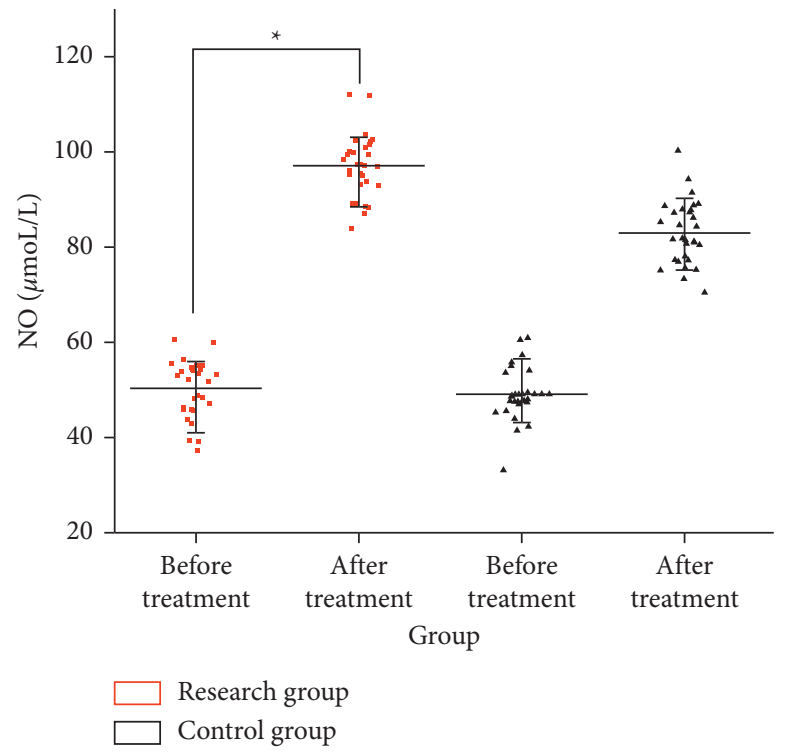

(a)

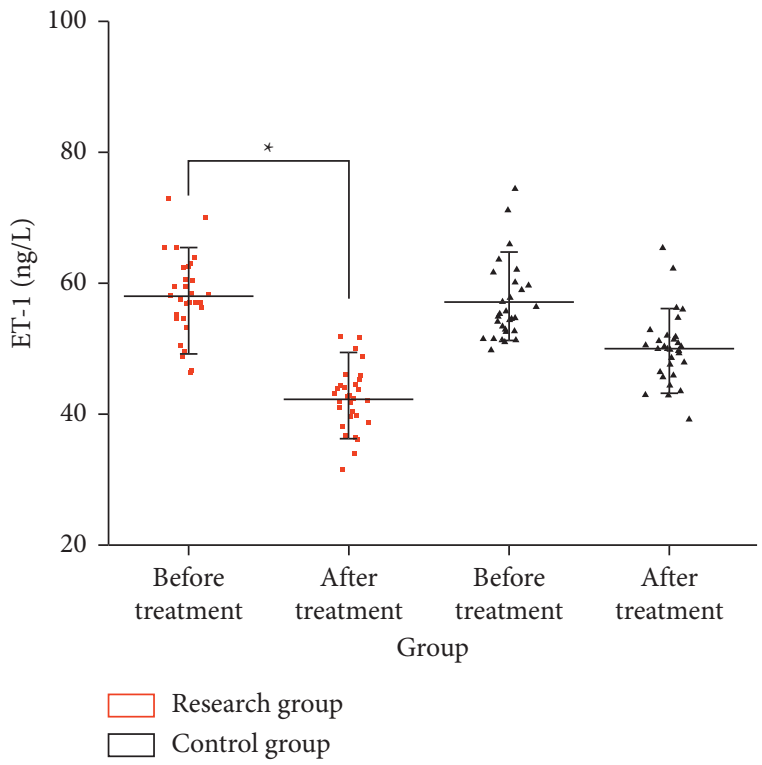

(b)

FIgure 2: Comparison of plasma NO and ET-1 levels before and after treatment in the two groups. (a) NO level and (b) ET-1 level.

a multifunctional zinc-dependent metalloprotease. Enkephalinase can inactivate some peptide hormones, including brain natriuretic peptide and bradykinin, vasodilator peptides, glucagon, substance $\mathrm{P}$, neurotensin, and oxytocin [23]. Valsartan is an angiotensin receptor antagonist. The main effect of valsartan is to inhibit the activation of the reninangiotensin-aldosterone system and improve the long-term prognosis of patients with heart failure. As a compound preparation, sacubitril valsartan can be used in heart failure with reduced ejection fraction, which can effectively improve 


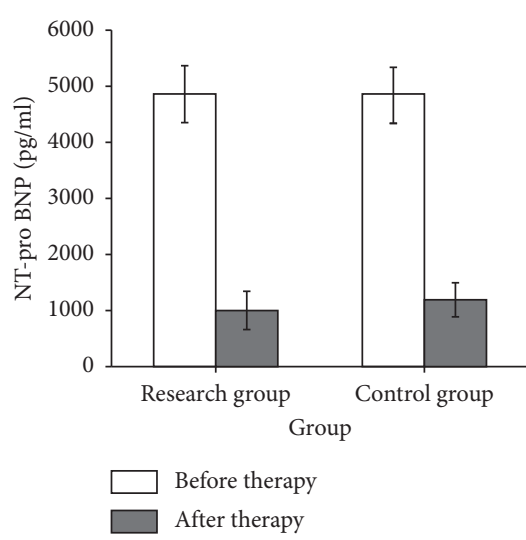

(a)

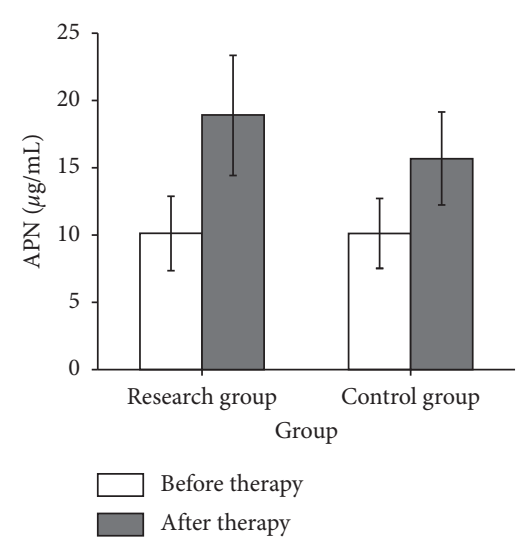

(b)

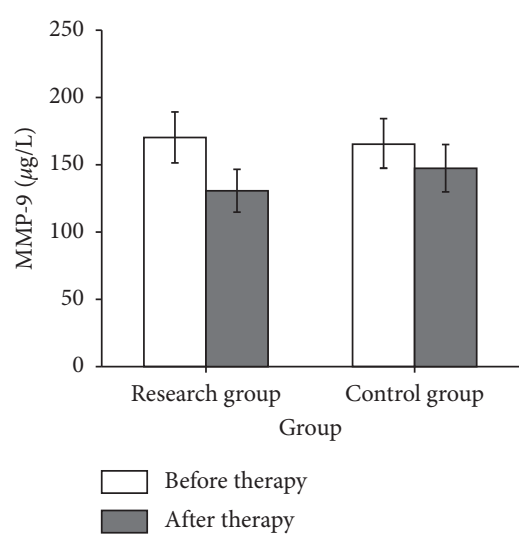

(c)

Figure 3: Comparison of NT-pro BNPAPN and MMP-9 levels before and after treatment in the two groups. Note. (a) Level of NT-pro BNP, (b) level of APN, and (c) level of MMP-9.

TABle 3: Comparison of carotid artery intima-media thickness before and after treatment between the two groups of patients, glomerular filtration rate, and left ventricular ejection fraction.

\begin{tabular}{|c|c|c|c|c|c|c|c|c|c|}
\hline \multirow{2}{*}{ Group } & \multicolumn{3}{|c|}{$\begin{array}{c}\text { Carotid artery intima-media } \\
\text { thickness }(\mathrm{mm})\end{array}$} & \multicolumn{3}{|c|}{ Glomerular filtration rate $(\mathrm{ml} / \mathrm{min})$} & \multicolumn{3}{|c|}{ Left ventricular ejection fraction (\%) } \\
\hline & $\begin{array}{c}\text { Before } \\
\text { treatment }\end{array}$ & $\begin{array}{c}\text { After } \\
\text { treatment }\end{array}$ & Difference & $\begin{array}{c}\text { Before } \\
\text { treatment }\end{array}$ & $\begin{array}{c}\text { After } \\
\text { treatment }\end{array}$ & Difference & $\begin{array}{c}\text { Before } \\
\text { treatment }\end{array}$ & $\begin{array}{c}\text { After } \\
\text { treatment }\end{array}$ & Difference \\
\hline $\begin{array}{l}\text { Control } \\
\text { group }\end{array}$ & $1.23 \pm 0.22$ & $1.28 \pm 0.24$ & $0.05 \pm 0.07$ & $123.45 \pm 19.35$ & $118.75 \pm 16.36$ & $-3.11 \pm 7.35$ & $39.39 \pm 1.81$ & $41.22 \pm 2.08$ & $1.83 \pm 0.66$ \\
\hline $\begin{array}{l}\text { Research } \\
\text { group }\end{array}$ & $1.24 \pm 0.21$ & $1.30 \pm 0.26$ & $0.05 \pm 0.09$ & $122.33 \pm 19.14$ & $121.86 \pm 17.83$ & $-0.47 \pm 5.15$ & $39.02 \pm 1.73$ & $41.44 \pm 2.11$ & $2.42 \pm 0.73$ \\
\hline
\end{tabular}

myocardial remodeling, thereby effectively reducing the risk of cardiovascular death in patients with heart failure, the risk of heart failure hospitalization, and the risk of all-cause death, and significantly improve and improve the symptoms and quality of life of patients. Studies have shown that sacubitril valsartan is an antihypertensive drug [24-26]. Sacubitril valsartan has a highly selective inhibition of enkephalinase, blocks angiotensin receptors, and plays an important role in improving heart failure, reducing ejection fraction, and controlling blood pressure $[15,27,28]$. In the occurrence and development of CHF, its vascular endothelial dysfunction and the disease itself are mutually causal. Brachial artery EDD is currently used clinically to evaluate the endothelial function of the body [29]. ET-1 is currently recognized as the strongest arteriovenous vasoconstrictor peptide, which is mainly secreted by endothelial cells. ET-1 can regulate vascular tone and stimulate the proliferation of vascular smooth muscle. The secretion of ET-1 in vivo increases when cardiomyocytes are damaged. Studies have shown that its expression is closely related to the severity of heart failure $[30,31]$. Endogenous NO can expand the tube, lower blood pressure and protect cardiomyocytes [32]. BNP is a polypeptide hormone secreted and synthesized by cardiomyocytes, and its content is very small in normal humans. When the heart load increases or the heart function is impaired, its plasma concentration increases. Therefore, $\mathrm{BNP}$ is a specific indicator for diagnosing chronic heart failure, and it has important clinical value in evaluating therapeutic efficacy and predicting prognosis. APN is a protective plasma hormone protein secreted by adipocytes. APN participates in the occurrence, development, and prognosis of chronic heart failure of coronary heart disease through mechanisms such as inhibiting vascular inflammation, inhibiting monocyte adhesion, and the formation of fibrous tissue plaques. Pathological ventricular remodeling is an important pathological change in cardiovascular diseases, and MMP-9 is the main protease involved in ventricular remodeling. Studies have shown that MMP-9 activity is related to the severity of coronary heart disease and chronic heart failure. The higher the serum content of MMP-9, the more serious the deterioration of heart function.

The research group selected valsartan capsules and sacubitril and valsartan sodium tablets for comprehensive treatment and nursing of chronic heart failure and hypertension. The results of the study showed that compared with the control group, which was treated with valsartan capsules alone, after treatment, the serum APN, EDD function, and NO levels of the two groups of patients were significantly higher than before treatment, and the levels of MMP-9, BNP, and ET-1 were lower than before treatment. The improvement of the study group was better than that of the control group $(P<0.05)$. The results show that sacubitril valsartan and valsartan can improve the levels of heart failure markers in patients with chronic heart failure to the 
greatest extent, protect cardiomyocytes, and delay the development of ventricular remodeling [33]. However, due to the limited number of patients participating in the study, the data have limitations.

In summary, the treatment of valsartan capsules and sacubitril and valsartan sodium tablets in patients with hypertensive $\mathrm{CHF}$ can improve the patient's vascular endothelial function, increase serum APN levels, reduce MMP9 and BNP levels, and improve the treatment efficacy of patients, which is worthy of clinical application.

\section{Data Availability}

The data to used support the findings of this study are available on reasonable request from the corresponding author.

\section{Conflicts of Interest}

The authors have no conflicts of interest to declare.

\section{References}

[1] M. Saitoh, M. R. Dos Santos, A. Emami et al., "Anorexia, functional capacity, and clinical outcome in patients with chronic heart failure: results from the Studies Investigating Co-morbidities Aggravating Heart Failure (SICA-HF)," Esc Heart Failure, vol. 4, no. 4, pp. 448-457, 2017.

[2] A. Chandra, E. F. Lewis, B. L. Claggett et al., "Effects of sacubitril/valsartan on physical and social activity limitations in patients with heart failure: a secondary analysis of the PARADIGM-HF trial," JAMA Cardiology, vol. 3, no. 6, pp. 498-505, 2018.

[3] B. Pieske, A. P. Maggioni, C. S. P. Lam et al., "Vericiguat in patients with worsening chronic heart failure and preserved ejection fraction: results of the SOluble guanylate Cyclase stimulatoR in heArT failurE patientS with PRESERVED EF (SOCRATES-PRESERVED) study," European Heart Journal, vol. 38, no. 15, pp. 1119-1127, 2017.

[4] M. A. Mamas, M. Sperrin, M. C. Watson et al., "Do patients have worse outcomes in heart failure than in cancer? A primary care based cohort study with 10 year follow up in Scotland," European Journal of Heart Failure, vol. 19, no. 9, pp. 1095-1104, 2017.

[5] C. Penna, F. Tullio, S. Femminò et al., "Obestatin regulates cardiovascular function and promotes cardioprotection through the nitric oxide pathway," Journal of Cellular and Molecular Medicine, vol. 21, no. 12, pp. 3670-3678, 2017.

[6] M. Cwynar, J. Gąsowski, T. Stompór et al., "Blood pressure and arterial stiffness in patients with high sodium intake in relation to sodium handling and left ventricular diastolic dysfunction status," Journal of Human Hypertension, vol. 29, no. 10, pp. 583-591, 2015.

[7] M. Yildiz, A. A. Oktay, M. H. Stewart, R. V. Milani, H. O. Ventura, and C. J. Lavie, "Left ventricular hypertrophy and hypertension," Progress in Cardiovascular Diseases, vol. 63, no. 1, pp. 10-21, 2020.

[8] T. G. Lueder and K. Henry, "RAAS inhibitors and cardiovascular protection in large scale trials," Cardiovascular Drugs and Therapy, vol. 27, no. 2, pp. 171-179, 2013.

[9] Z. Sameer, S. S. Zaman, S. Timothy et al., "The mortality risk of deferring optimal medical therapy in heart failure: a systematic comparison against norms for surgical consent and patient information leaflets," European Journal of Heart Failure, vol. 19, no. 11, pp. 1401-1409, 2017.

[10] T. G. von Lueder, S. J. Sangaralingham, B. H. Wang et al., "Renin-angiotensin blockade combined with natriuretic peptide system Augmentation," Circulation: Heart Failure, vol. 6, no. 3, pp. 594-605, 2013.

[11] H.-M. Choi and M.-S. Shin, “Angiotensin receptor-neprilysin inhibitor for the treatment of heart failure: a review of recent evidence," The Korean Journal of Internal Medicine, vol. 35, no. 3, pp. 498-513, 2020.

[12] M. Senni, J. J. V. McMurray, R. Wachter et al., "Initiating sacubitril/valsartan (LCZ696) in heart failure: results of TITRATION, a double-blind, randomized comparison of two uptitration regimens," European Journal of Heart Failure, vol. 18, no. 9, pp. 1193-1202, 2016.

[13] M. Juan Tamargo, "The mechanism of action of LCZ696," Cardiac Failure Review, vol. 2, no. 1, p. 40, 2016.

[14] V. Orly, C. Brian, K. Jessica et al., "Incidence, predictors, and outcomes associated with hypotensive episodes among heart failure patients receiving sacubitril/valsartan or enalapril: the PARADIGM-HF trial (prospective comparison of AnpritensinReceptor with angiotensin-converting enzyme inhibitor to determine impacton global mortality and morbidity in heart failure)," Circulation: Heart Failure, vol. 11, no. 4, Article ID e004745, 2018.

[15] R. De Vecchis, C. Ariano, G. Di Biase, and M. Noutsias, "Sacubitril/valsartan for heart failure with reduced leftventricular ejection fraction: a retrospective cohort study," Herz, vol. 44, no. 5, pp. 425-432, 2019.

[16] M. Böhm, R. Young, P. S. Jhund et al., "Systolic blood pressure, cardiovascular outcomes and efficacy and safety of sacubitril/valsartan (LCZ696) in patients with chronic heart failure and reduced ejection fraction: results from PARADIGM-HF," European Heart Journal, vol. 38, no. 15, pp. 1132-1143, 2017.

[17] L. M. Ruilope, A. Dukat, M. Böhm, Y. Lacourcière, J. Gong, and M. P. Lefkowitz, "Blood-pressure reduction with LCZ696, a novel dual-acting inhibitor of the angiotensin II receptor and neprilysin: a randomised, double-blind, placebo-controlled, active comparator study," Lancet, vol. 375, no. 9722, pp. 1255-66, 2010.

[18] A. Mazza, D. M. Townsend, G. Torin et al., "The role of sacubitril/valsartan in the treatment of chronic heart failure with reduced ejection fraction in hypertensive patients with comorbidities: from clinical trials to real-world settings," Biomedicine \& Pharmacotherapy, vol. 130, Article ID 110596, 2020.

[19] C. W. Yancy, M. Jessup, B. Bozkurt et al., “ACCF/AHA guideline for the management of heart failure: executive summary: a report of the American College of Cardiology Foundation/American Heart Association Task Force on practice guidelines," Circulation, vol. 128, no. 16, pp. 1810-1852, 2013.

[20] J. J. McMurray, S. Adamopoulos, S. D. Anker et al., "ESC guidelines for the diagnosis and treatment of acute and chronic heart failure 2012: the task force for the diagnosis and treatment of acute and chronic heart failure 2012 of the European society of cardiology. Developed in collaboration with the heart failure association (HFA) of the ESC," European Heart Journal, vol. 33, no. 14, pp. 1787-1847, 2012.

[21] D. Tomasoni, M. Adamo, M. S. Anker, S. Haehling, A. J. S. Coats, and M. Metra, "Heart failure in the last year: progress and perspective," ESC Heart Failure, vol. 7, no. 6, pp. 3505-3530, 2020. 
[22] B. N. Reed, S. E. Street, and B. C. Jensen, "Time and technology will tell," Heart Failure Clinics, vol. 10, no. 4, pp. 543-557, 2014.

[23] P. S. Jhund and J. J. V. McMurray, "The neprilysin pathway in heart failure: a review and guide on the use of sacubitril/ valsartan," Heart, vol. 102, no. 17, pp. 1342-1347, 2016.

[24] K. Kario, Y. Tamaki, N. Okino, H. Gotou, M. Zhu, and J. Zhang, "LCZ696, a first-in-class Angiotensin receptorneprilysin inhibitor: the first clinical experience in patients with severe hypertension," Journal of Clinical Hypertension, vol. 18, no. 4, pp. 308-314, 2016.

[25] M. B. Andersen, U. Simonsen, M. Wehland, J. Pietsch, and D. Grimm, "LCZ696 (Valsartan/Sacubitril) - a possible new treatment for hypertension and heart failure," Basic and Clinical Pharmacology and Toxicology, vol. 118, no. 1, pp. 14-22, 2016.

[26] G. Ruiz-Hurtado and L. M. Ruilope, "Advantages of sacubitril/valsartan beyond blood pressure control in arterial hypertension," European Heart Journal, vol. 38, no. 44, pp. 3318-3320, 2017.

[27] L. Zheng, B. Xia, X. Zhang, and Y. Zhao, "A meta-analysis on the effect and safety of LCZ696 in the treatment of hypertension," Cardiology Research and Practice, vol. 2021, Article ID 8867578, 2021.

[28] J. Gu, A. Noe, P. Chandra et al., "Pharmacokinetics and pharmacodynamics of LCZ696, a novel dual-acting angiotensin receptor-neprilysin inhibitor (ARNi)," The Journal of Clinical Pharmacology, vol. 50, no. 4, pp. 401-414, 2010.

[29] T. Maruhashi, Y. Iwamoto, M. Kajikawa et al., K. Noma, A. Nakashima, C. Goto, E. Hida, and Y. Higashi, "Interrelationshipsamong flow-mediated vasodilation, nitroglycerine-induced vasodilation, baseline brachial artery diameter, hyperemicshear stress, and cardiovascular risk factors," Am Heart Association, vol. 7, no. 1, pp. e797-e803, 2018.

[30] A. H. Chester and M. H. Yacoub, "The role of endothelin-1 in pulmonaryarterial hypertension," Glob Cardiol Sci Pract, vol. 2014, no. 2, pp. 62-78, 2017.

[31] Q. Wu, Y. Cao, G. Mao et al., "Effects of forest bathing on plasma endothelin-1 in elderly patients with chronic heart failure: implications for adjunctive therapy," Geriatrics and Gerontology International, vol. 17, no. 12, pp. 2627-2629, 2017.

[32] G. P. Rossi, T. M. Seccia, M. Barton et al., "Endothelial factors in the pathogenesis and treatment of chronic kidney disease part I: general mechanisms a joint consensus statement from the European society of hypertension working group onendothelin and endothelial factors and the Japanese society," Hypertension, vol. 36, no. 3, pp. 1-10, 2018.

[33] A. Zimmer, R. B. Teixeira, J. H. Bonetto et al., "Effects of aerobic exercise training on metabolism of nitric oxide and endothelin-1 in lung parenchyma of rats with pulmonary arterial hypertension," Molecular and Cellular Biochemistry, vol. 429, no. 2, pp. 73-89, 2017. 